\title{
Preparation and Evaluation of Chitosan Microcapsules of Metronidazole using Tripolyphosphate Cross-linking Method
}

\author{
Akanksha Garud* and Navneet Garud
}

\author{
Department of Pharmaceutics, Institute of Professional Studies-College of Pharmacy, \\ Gwalior 474002 (M.P.), India
}

\begin{abstract}
Metronidazole microcapsules with a coat consisting of alginate and the natural cationic polymer, chitosan were formulated by using tripolyphosphate cross-linking method and were investigated with a view to develop mucoadhesive microcapsules. The microcapsules were evaluated for their surface morphology, microencapsulation efficiency, in-vitro wash-off test, swelling behavior and in-vitro drug release. The microcapsules formed had rough surface morphology in scanning electron microscopy. The drug entrapment efficiency was found to be in the range $75.2 \pm 1.31 \%$ and $82.1 \pm 0.75 \%$. Chitosan microcapsules displayed a limited amount of swelling which is supposed to be related to the degree of cross-linking with tripolyphosphate. The microcapsules showed better mucoadhesive property at intestinal $\mathrm{pH} 7.4$ than at gastric $\mathrm{pH} 1.2$ in the in-vitro wash-off test. The drug release was found to be slow and extended over long duration of time.
\end{abstract}

Key words: Metronidazole microcapsules, chitosan, mucoadhesion, tripolyphosphate

\section{INTRODUCTION}

Amoebiasis is an important cause of death by protozoal infections, next only to malaria in the developing countries; even in advanced countries it has emerged as an important infection among imunosuppressed individuals. Metronidazole (Mtz) has extremely broad spectrum of protozoal and antimicrobial activity. It is clinically effective in trichomoniasis, amoebic colitis and giardiasis. The drug has made an important contribution to the management of amoebiasis. ${ }^{1}$

Microencapsulation has been accepted as a process to achieve controlled release and drug targeting. Microspheres constitute an important part of these particulate DDS by virtue of their small size and efficient carrier characteristics. However, the

Correspondence to:

Akanksha Garud

E-mail: akanksha.garud@gmail.com

Dhaka Univ. J. Pharm. Sci. 9(2): 125-130, 2010 (December) success of these novel DDS is limited due to their short residence time at the site of absorption. It would, therefore, be advantageous to have means for providing an intimate contact of the DDS with the absorbing membranes, which can be achieved by coupling bioadhesion characteristics to microspheres and developing novel delivery systems referred to as "bioadhesive microspheres". Mucoadhesion prolongs the residence time of the dosage form at the site of application and thus results in improving and enhancing the bioavailability of the drug. ${ }^{2-4}$

Among different approaches to prepare oral controlled release mucoadhesive microcapsules, the use of natural polymer chitosan has gained much importance. Chitosan is obtained by N-alkaline deacetylation of chitin present in the shells of crustaceans, molluscs, the cell walls of fungi, and the cuticle of insects. It is a cationic linear polysaccharide composed essentially of $\beta(1 \rightarrow 4)$ linked glucosamine units together with some 
proportion of $\mathrm{N}$-acetyl-glucosamine units. Chitosan is a biocompatible, biodegradable, non-toxic and mucoadhesive polymer; which makes it attractive for applications in medicine and pharmacy. The mucoadhesive property of chitosan is due to its positive charge at neutral $\mathrm{pH}$ resulting in an ionic interaction with the negative charges of sialic acid residues of the mucus. The natural polymer chitosan and sodium alginate; a cationic and an anionic agent, respectively have received particular attention ${ }^{5-9}$.

The present study focuses on the formulation and evaluation of mucoadhesive microcapsules containing metronidazole using different concentration of the naturally occurring cationic polymer chitosan (1\% to $6 \%$ ); cross-linked with tripolyphosphate, using sodium alginate designed for oral controlled release. Metronidazole has side effects like nausea, abdominal cramps, metallic taste and half-life of 6 to $8 \mathrm{hr}$ which initiated the need to be formulated as microcapsules.

\section{MATERIALS AND METHODS}

Metronidazole was a gift sample from J.B.Chemicals and Pharmaceuticals Limited, Ankleshwar. Chitosan (medium viscosity grade) was a gift sample from Central Institute of Fisheries Technology, Cochin. Sodium alginate (S.D.Fine Chemicals, Ltd.; Boisar) and Tripolyphosphate (TPP), Loba Chemicals were purchased. All other chemicals used were of analytical grade.

Preparation of mucoadhesive microcapsules ${ }^{10-}$ 12. About $200 \mathrm{mg}$ of metronidazole and $100 \mathrm{ml}$ of chitosan solution in different concentrations (1\% to $4 \%)$ of $0.1 \mathrm{M}$ acetic acid $(1.0 \% \mathrm{w} / \mathrm{v})$ were mixed together to obtain drug-chitosan solution. About $2 \mathrm{~g}$ of sodium alginate was dissolved in $100 \mathrm{ml}$ distilled water and mixed for approximately four hours. The hybrid microcapsules were prepared according to the following scheme. First, calcium alginate beads were prepared by dropping the alginate solution through a needle into a $0.5 \mathrm{M}$ calcium chloride solution. After 5 min. of cross-linking, the beads were taken out and washed. The beads were then suspended in the drug- chitosan solution; the hybrid microcapsules were formed by taking the beads into a specially modified plastic pipette with a slight suction, and dropping it into sodium tripolyphosphate, TPP ( $2 \%$ and $4 \% \mathrm{w} / \mathrm{v})$ solution. The microcapsules were kept in TPP solution (cross-linked solution) for 2 hrs and washed with distilled water.

Estimation of Metronidazole. Metronidazole content in the microcapsules was estimated by using U.V.Spectrophotometer (Shimadzu 1700) method based on the measurement of absorbance at $278 \mathrm{~nm}$ in $0.1 \mathrm{~N} \mathrm{HCl}$ and at $320 \mathrm{~nm}$ in $\mathrm{pH} 7.4$ Phosphate Buffer. The method obeyed Beer's law in the concentration range from 2 to $20 \mu \mathrm{g} / \mathrm{ml}$.

Morphology. Shape and surface morphology of chitosan-alginate hybrid microcapsules were studied using Scanning Electron Microscope (SEM, JSM 6100, Jeol, Japan). Particle sizes of the microcapsules were evaluated using optical microscope.

Microencapsulation efficiency. An appropriate amount of microcapsules were first crushed and then weighed and suspended in methanol to extract the drug from the microcapsules. After $24 \mathrm{~h}$, the filtrate was assayed spectrophotometrically (Shimadzu 1700) at $320 \mathrm{~nm}$ for drug content. The microencapsulation efficiency was calculated using the formula ${ }^{4,13}$ :

Microencapsulation efficiency $=($ Practical drug content/ Theoretical drug content) X 100

Determination of swelling behavior. The water-sorption capacity of the metronidazole microcapsules were determined by swelling $200 \mathrm{mg}$ of dry microcapsules in phosphate-buffered saline (PBS) $\mathrm{pH} 7.4$ for at least 24 hrs. The wet weight of the swollen microcapsules was determined by first blotting the microcapsules with filter paper to remove surface water and then weighed immediately. The percentage swelling of the metronidazole microcapsules were calculated as ${ }^{14}$ :

$$
\mathrm{S}=(\text { We }- \text { Wo }) / \text { Wo } \times 100 \%
$$

where, We denotes the weight of the gel microcapsules at equilibrium swelling and Wo the initial weight of the microcapsules. 
In vitro wash-off test. The mucoadhesive property of the microcapsules was evaluated by the in-vitro wash-off test ${ }^{15,16}$ or in vitro adhesion testing method. The mucoadhesiveness of these microcapsules was compared with non-bioadhesive material, ethylene vinyl acetate microcapsules. Pieces of intestinal mucosa $(2 \mathrm{x} 2 \mathrm{~cm}$.) were mounted on to glass slides (3x1 inch.) with cyanoacrylate glue. Two glass slides were connected with a suitable support. About 50 microcapsules were spread on to each wet rinsed tissue specimen and immediately thereafter the support was hung on to the arm of a U.S.P. tablet disintegrating test machine. A slow regular up and down moment was given in a test fluid at $37^{\circ} \mathrm{C}$ in $1 \mathrm{~L}$ vessel of the machine. At the end of $30 \mathrm{~min}, 1 \mathrm{~h}$ and later at hourly intervals upto $12 \mathrm{hrs}$, the machine was stopped and the number of microcapsules still adhering on to the tissue was counted. The test was performed in $0.1 \mathrm{~N} \mathrm{HCl}$ and in phosphate buffer of $\mathrm{pH} 7.4$.

In vitro drug release. The in vitro drug release ${ }^{17}$ profiles from various formulations of microspheres (about $500 \mathrm{mg}$ ) were studied in $900 \mathrm{ml}$ of buffer with gastrointestinal simulated $\mathrm{pH}$ conditions, viz. simulated gastric fluid $(0.1 \mathrm{~N} \mathrm{HCl}, \mathrm{pH} 1.2)$ for first 2 hours followed by next 4 hours in simulated intestinal fluid (phosphate buffer solution, PBS, pH 6.8) and finally up to $15 \mathrm{~h}$ in simulated intestinal fluid (phosphate buffer solution, PBS, pH 7.4) at $100 \mathrm{rpm}$. At different time intervals, $10 \mathrm{ml}$. of samples were withdrawn and filtered through a $0.8 \mu \mathrm{m}$ cellulose acetate membrane filter and estimated for metronidazole concentration at $320 \mathrm{~nm}$ using Shimadzu, Pharmspec UV-1700 series, Japan.

Statistical analysis. Experimental data have been represented as the mean with standard deviation (SD) of different independent determinations. The significance of differences was evaluated by analysis of variance (ANOVA). Differences were considered statistically significant at $\mathrm{P}<0.05$.

\section{RESULTS AND DISCUSSION}

Morphology. The polymer surface of the microcapsules as observed by SEM was heterogeneous and porous. These findings were similar with those of other researchers who reported rough surface morphologies for chitosan microspheres prepared by the orifice-ionic gelation technique. $^{18,19}$ However, Kumbar et al., 2002 reported smooth surface morphologies of chitosan microspheres when chitosan microspheres were produced in w/o emulsion and then cross-linked with glutaraldehyde. The surface morphology characteristics have an impact on bioadhesion. It has been found that the microspheres with a coarser and more porous surface may offer enhanced bioadhesivity as compared to those with a smoother texture $^{2}$. Rough coarse surface observed in the present study might have led to bioadhesion. The surface morphology show the formation of cracks on the surface of the microcapsules which may be due to the penetration of the dissolution medium into the microcapsules and the subsequent dissolution of the drug and hence its diffusion through the polymer matrix (Figure 1).

Drug loading efficiency. The microencapsulation efficiency for the different formulations was found to be in the range of $75.2 \pm 1.31 \%$ to $82.1 \pm 0.75 \%$. The encapsulation efficiency increased markedly with increasing chitosan content. The mean particle size ranged between $2.1 \pm 0.36 \mathrm{~mm}$ and $2.9 \pm 0.11 \mathrm{~mm}$. Longer the cross-linking time or the cross-linker concentration, the smaller was the average size of the microcapsules (Table 1).

Swelling behavior. Swelling behavior of chitosan microcapsules in phosphate buffer of $\mathrm{pH} 7.4$ was studied. It can be observed that chitosan microcapsules displayed a limited amount of swelling with a maximum degree of swelling achieved after 2 $\mathrm{h}$ of exposure to the dissolution medium. This minimal swelling may be attributed to the reduced solubility of chitosan at alkaline $\mathrm{pH}$ and consequently the inability to absorb water and swell ${ }^{9,19,20}$. In addition, the limited amount of swelling may have been related to the degree of cross linking with tripolyphosphate which can be attributed to the 
gelling mechanism of the metronidazole microcapsules (Table 1).

In vitro wash-off test. The in-vitro wash-off test showed that as the percentage of chitosan is increased in the formulation, its mucoadhesiveness increases. The wash off was faster at intestinal pH 7.4 than at gastric $\mathrm{pH}$ 1.2. The results of the wash-off test indicated that the microcapsules had fairly good mucoadhesive property. At a higher concentration of chitosan coiling of the polymer molecules occur, reducing the flexibility of the polymeric chain, thereby reducing the bioadhesive strength. At lower chitosan concentration, the polymer structure of the microcapsules is looser and polymer chains thus have more space to extend within the mucin. ${ }^{19}$ The percentage of microcapsules adhering to tissue at $\mathrm{pH}$ 7.4 after $12 \mathrm{~h}$ varied from 8 to 20, whereas the percentage of microcapsules adhering to tissue at $\mathrm{pH}$ 1.2 after $12 \mathrm{~h}$ varied from 12 to 39 . (Table 2 )

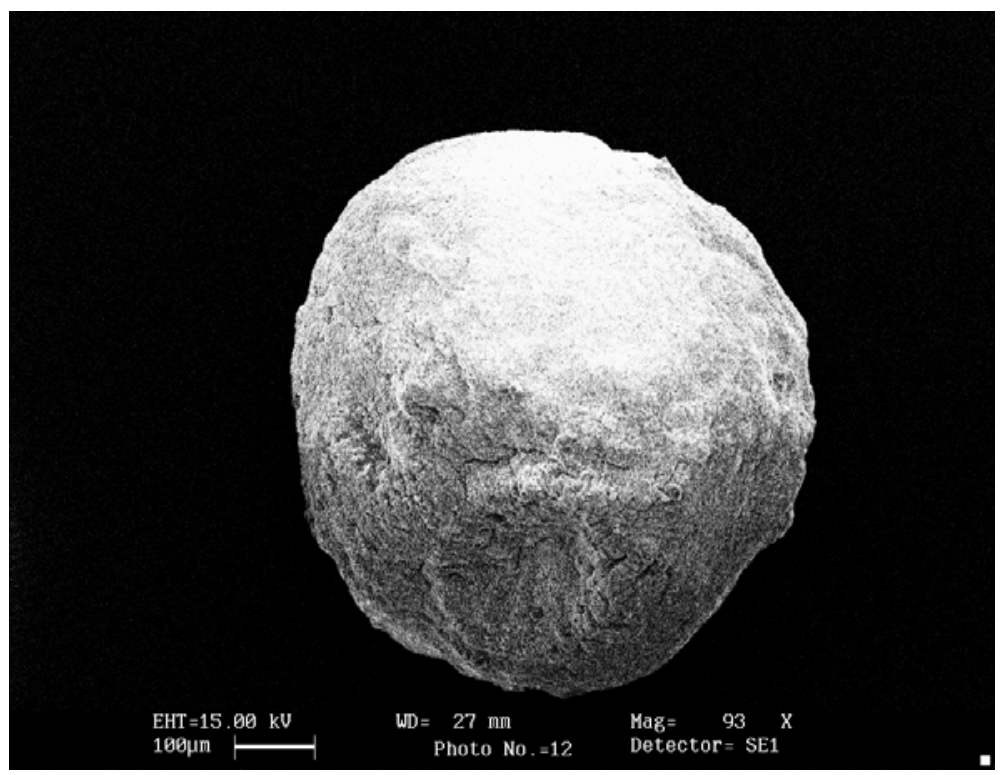

Figure 1. Scanning electron micrograph of metronidazole microcapsules for formulation C4

Table 1. Composition and characteristics of different Chitosan microcapsules.

\begin{tabular}{llllllll}
\hline $\begin{array}{c}\text { Batch } \\
\text { code }\end{array}$ & $\begin{array}{c}\text { Chitosan } \\
(\% \mathrm{w} / \mathrm{v})\end{array}$ & $\begin{array}{c}\text { Sodium } \\
\text { alginate } \\
(\% \mathrm{w} / \mathrm{w})\end{array}$ & $\begin{array}{c}\text { TPP } \\
(\% \mathrm{w} / \mathrm{v})\end{array}$ & $\begin{array}{c}\text { Cross- } \\
\text { linking } \\
\text { time }(\mathrm{min})\end{array}$ & $\begin{array}{c}\text { Degree of } \\
\text { swelling* }\end{array}$ & $\begin{array}{c}\text { Average } \\
\text { particle size } \\
(\mathrm{mm})( \pm \mathrm{SD}) *\end{array}$ & $\begin{array}{c}\text { Microencapsulation } \\
\text { efficiency }(\%) \\
( \pm \text { SD)* }\end{array}$ \\
\hline $\mathrm{C} 1$ & 1 & 2 & 2 & 10 & $0.78 \pm 0.31$ & $2.2 \pm 0.41$ & $75.2 \pm 1.31$ \\
$\mathrm{C} 2$ & 2 & 2 & 2 & 10 & $1.32 \pm 0.67$ & $2.4 \pm 0.20$ & $78.5 \pm 2.26$ \\
$\mathrm{C} 3$ & 3 & 2 & 2 & 10 & $1.44 \pm 0.85$ & $2.7 \pm 0.23$ & $80.6 \pm 0.88$ \\
$\mathrm{C} 4$ & 4 & 2 & 2 & 10 & $1.54 \pm 1.12$ & $2.9 \pm 0.11$ & $82.1 \pm 0.75$ \\
$\mathrm{C5}$ & 4 & 2 & 4 & 10 & $1.16 \pm 0.76$ & $2.1 \pm 0.36$ & $80.4 \pm 1.14$ \\
$\mathrm{C6}$ & 4 & 2 & 4 & 20 & $0.88 \pm 0.42$ & $1.6 \pm 0.22$ & $78.8 \pm 0.52$ \\
\hline
\end{tabular}

*Data are expressed as mean $\pm \mathrm{SD}$ on triplicate.

In vitro release studies. The in-vitro release rate showed that increasing the amount of chitosan in the microcapsules decreased the release rate after $12 \mathrm{~h}$. The drug release from the microcapsules can further be controlled by cross linking of chitosan. It was observed that the amount of drug released from microcapsules decreased on increasing the cross linking time. A decrease of drug release was also 
observed with an increase in TPP concentration ( $\mathrm{P}<$ 0.05). Preparation of such systems involves the treatment of chitosan with reagents that cause formation of networks involving covalent or ionic bonds. Systems of these kinds are swellable in aqueous environment and have properties that depend on the density of cross-linking and the reagent used. The negatively charged tripolyphosphate reacted with the positively charged amine residues of chitosan to form ionic cross-links (Figure 2).

The bioadhesion, drug release, swelling behavior and other mentioned characterization data obtained in this study confirms the potential of the formulations C4, C5 and C6 to be effective in localizing the metronidazole drug to the specific site for treating the disease and maintaining the sustained effect of the formulation.

Table 2. Percentage of microcapsules adhering to tissue at different time intervals.

\begin{tabular}{|c|c|c|c|c|c|c|c|c|c|c|}
\hline \multirow{3}{*}{$\begin{array}{l}\text { Batch } \\
\text { Code }\end{array}$} & \multicolumn{10}{|c|}{$\%$ of microcapsules adhering to the tissue up to $12 \mathrm{~h}$} \\
\hline & \multicolumn{6}{|c|}{$0.1 \mathrm{~N} \mathrm{HCl}, \mathrm{pH} 1.2$} & \multicolumn{4}{|c|}{ Phosphate Buffer, pH 7.4} \\
\hline & 1 & 2 & 4 & 8 & 12 & 1 & 2 & 4 & 8 & 12 \\
\hline $\mathrm{C} 1$ & $72(1.2)$ & 59(1.5) & 51(0.8) & 39(1.8) & 12(1.5) & $52(2.1)$ & $39(2.4)$ & $28(0.7)$ & $20(0.8)$ & $08(1.1)$ \\
\hline $\mathrm{C} 2$ & $76(0.8)$ & $61(1.2)$ & $49(2.1)$ & $42(2.1)$ & $24(2.1)$ & $58(1.1)$ & $42(2.0)$ & $31(1.1)$ & $20(1.1)$ & $12(0.8)$ \\
\hline C3 & $81(0.7)$ & $74(0.8)$ & $66(2.6)$ & $51(0.8)$ & $28(0.8)$ & $66(0.8)$ & $57(1.2)$ & $44(0.8)$ & 26(1.6) & $10(2.1)$ \\
\hline $\mathrm{C} 4$ & $83(1.1)$ & $79(0.6)$ & $71(1.4)$ & $59(2.2)$ & $32(0.5)$ & $71(0.6)$ & $56(1.1)$ & $41(1.5)$ & $32(0.8)$ & $18(1.5)$ \\
\hline C5 & $85(0.6)$ & $79(1.2)$ & $72(0.8)$ & $63(1.5)$ & 39(1.4) & $75(1.2)$ & $58(1.5)$ & $36(2.1)$ & $22(1.5)$ & $12(2.0)$ \\
\hline C6 & $86(1.5)$ & $82(0.8)$ & $74(1.1)$ & $69(1.1)$ & $32(1.1)$ & $82(2.1)$ & $64(1.1)$ & $46(1.0)$ & $31(2.1)$ & $20(0.8)$ \\
\hline
\end{tabular}

Figures in parentheses are coefficient of variation

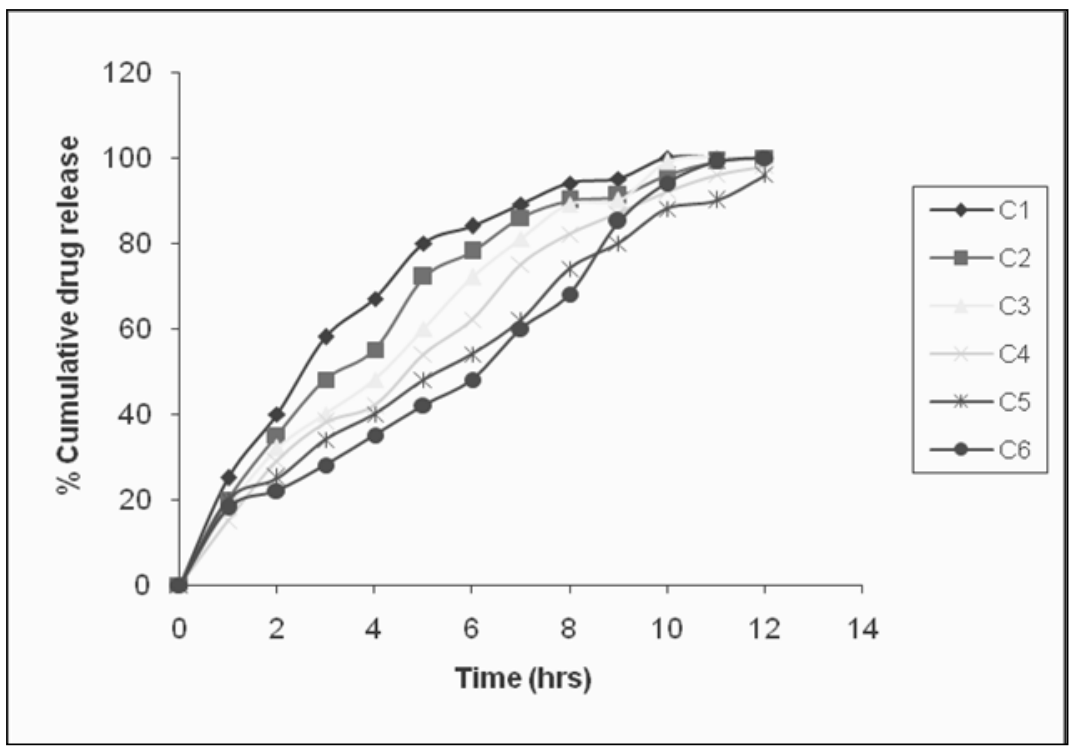

Figure 2 In vitro release profile of metronidazole mucoadhesive microcapsules

\section{ACKNOWLEDGEMENTS}

The authors thank J.B. Chemicals and Pharmaceuticals Limited, Ankleshwar for providing gift sample of metronidazole and Central Institute of Fisheries Technology, Cochin for providing gift sample of chitosan.

\section{REFERENCES}

1. Gupta, V., Rathi, A.K., Gupta, V.K. and Gupta, K. 1978. Therapeutic trial with syrup of metronidazole in amoebiasis. Indian J. Pediatrics. 45, 24-27.

2. Vasir, J.K., Tambwekar, K. and Garg, S. 2003. Bioadhesive microspheres as a Controlled drug delivery system, Int. J. Pharm. 255, 13-32. 
3. Chowdary, K.P.R. and Srinivasa, Y. 2003. Preparation and Evaluation of Mucoadhesive Microcapsules of Indomethacin. Ind. J. Pharrn. Sci. 65, 49-52.

4. Chowdary, K.P.R. and Rao, Y.S. 2003. Design and in vitro and in vivo evaluation of mucoadhesive microcapsules of glipizide for oral controlled release: A technical note. AAPS Pharm. Sci. Tech. 4, Article 39, 1-6.

5. Mishra, B., Jayanth, P. and Sarkar, C. 2003. Development of Chitosan-alginate microcapsules for colon specific delivery of Metronidazole. Indian Drugs. 40, 695-700.

6. Taqieddin, E., Lee, C. and Amiji, M. 2002. Perm-Selective Chitosan-Alginate Hybrid Microcapsules for Enzyme Immobilization Technology. Pharm. Eng. 22, 192-196.

7. Dhawan, S., Singla, A.K. and Sinha, V.R. 2004. Evaluation of mucoadhesive properties of chitosan microspheres prepared by different methods. AAPS Pharm. Sci. Tech. 5, Article 67.

8. Illum L. 1998. Chitosan and its use as a pharmaceutical excipients. Pharm. Res. 15, 1326-1331.

9. Singla, A.K. and Chawla, M. 2001. Chitosan: some pharmaceutical and biological aspects-an update. J. Pharm. Pharmacol. 53, 1047-1067.

10. Park, S.B., Kang, H.W., Haam, S., Park, H.Y. and Kim, W.S. 2004. Ca-alginate microspheres encapsulated in chitosan beads. J. Microencap. 21, 485-497.

11. Kumbar, S.G., Kulkarni, R. and Aminabhavi. 2002. Crosslinked Chitosan microspheres for encapsulation of Diclofenac sodium: effect of cross-linking agent. $J$. Microencap. 19, 173-180.

12. El-Gibaly, I. 2002. Development and in vitro evaluation of novel floating chitosan microcapsules for oral use: comparison with non-floating chitosan microspheres. Int.J.Pharm. 249, 7-21.
13. Prajapati, S.K., Tripathi, P., Ubaidulla, U. and Anand, V. 2008. Design and development of gliclazide mucoadhesive microcapsules: In vitro and in vivo evaluation. AAPS Pharm. Sci. Tech. 9, 224-230.

14. Shankar, N.B., Kumar, N.U. and Balakrishna, P.K. 2009. Formulation design, preparation and in vitro evaluation of Mucoadhesive microcapsule employing control release polymers to enhance gastroretention for oral delivery of Famotidine. Int. J. Pharm. Sci. Tech. 2, 22-29.

15. Chowdary, K.P.R., and Rao, Y.S. 2003. Mucoadhesive microcapsules of glipizide: characterization, in vitro and in vivo evaluation. Indian J. Pharm. Sci. 65, 279-284.

16. Singh, C., Jain, K.A., Kumar, C. and Agarwal, K. 2009. Design and in-vitro evaluation of mucoadhesive microcapsules of pioglitazone. J. Young Pharmacists. 1, 195198.

17. Nayak, B.S., Ghosh, S.K. and Patro, K.T.B. 2009. Preparation and characterization of famotidine microcapsule employing Mucoadhesive polymers in combination to enhance gastroretention for oral delivery, IJPPS. 1, 112-120.

18. Shu, X.Z. and Zhu, K.J. 2000. A Novel approach to prepare tripolyphosphate / chitosan complex beads for controlled drug delivery. Int. J. Pharm. 201, 51-58.

19. Govender, S., Pillay, V., Chetty, D.J. and Essack, S. Y. 2005 Optimization and characterization of bioadhesive controlled release tetracycline microspheres. Int. J. Pharm. 306, 24-40.

20. Lin, W.C., Yu, D.G. and Yang, M.C. 2005. pH-sensitive polyelectrolyte complex gel microspheres composed of Chitosan/sodium tripolyphosphate/dextran sulphate: swelling kinetics and drug delivery properties. Colloids and Surfaces B: Biointerfaces, 44, 143-157. 